\title{
Search for lepton flavour violating decays at $\mathrm{LHCb}$
}

\section{Marco Santimaria* $\dagger$}

INFN - Laboratori Nazionali di Frascati, Italy

E-mail: marco.santimaria@cern.ch

Several measurements on $b$-hadron decays suggest a deviation from the Lepton Flavour Universality property of the Standard Model. New Physics models have been proposed to coherently explain these alleged deviations by introducing non-universal interactions mediated for example by $Z^{\prime}$ bosons or leptoquarks. Since these non-universal lepton flavour couplings usually imply the non-conservation of the lepton flavour, searches for these processes are pursued by the LHCb experiment. Three recent results in this field are reported in this document: $B_{(s)}^{0} \rightarrow e^{+} \mu^{-}$, $B_{(s)}^{0} \rightarrow \tau^{+} \mu^{-}$and $B^{+} \rightarrow K^{+} e^{ \pm} \mu^{\mp}$ searches with $3 \mathrm{fb}^{-1}$ of data.

European Physical Society Conference on High Energy Physics - EPS-HEP2019 -

10-17 July, 2019

Ghent, Belgium

* Speaker.

${ }^{\dagger}$ On behalf of the LHCb collaboration. 


\section{Introduction}

Although the conservation of lepton flavour is experimentally well established for charged particles [1, 2, 3], the Standard Model (SM) lacks of the corresponding symmetry. The oscillation of neutrinos proves that Lepton Flavour Violation (LFV) processes do occur in nature, so that charged LFV can also be mediated at one-loop, yet at unmeasurably low rates $\left(\approx 10^{-54}\right)$ due to the smallness of the neutrino masses [4]. Evidence of charged LFV decays would therefore be an unambiguous sign of New Physics (NP).

Moreover, several experimental measurements of Lepton Flavour Universality (LFU) in $b$-hadron decays show discrepancies with respect to the SM expectations to a degree that demands further investigation [5]. NP models have been proposed to accommodate simultaneously these alleged deviations via non-universal interactions, mediated for example by additional gauge bosons $\left(Z^{\prime}[6]\right)$ or leptoquarks (LQ [7]). If non-universal leptonic interactions are allowed, a violation of lepton flavour conservation is necessarily implied [8]. Under certain couplings and mediator masses, these NP models predict enhancements of LFV process rates up to levels accessible at the LHC.

The above considerations strongly motivate the search for LFV processes: if no signal is observed, upper limits on the branching fractions are useful to constrain NP parameters and ultimately help to shed light on LFU.

The LHCb detector [9] is especially suited to search for LFV processes in $b$-hadron decays, thanks to its large $b \bar{b}$ acceptance, high vertex resolution and efficient muon trigger. Three recent searches will be reported in this document: $B_{(s)}^{0} \rightarrow e^{+} \mu^{-}(\text {Sec. 2) })^{1}, B_{(s)}^{0} \rightarrow \tau^{+} \mu^{-}$(Sec. 3) and $B^{+} \rightarrow$ $K^{+} e^{ \pm} \mu^{\mp}$ (Sec. 4). All the analyses are performed using a dataset corresponding to an integrated luminosity of $3 \mathrm{fb}^{-1}$ collected by the LHCb experiment in 2011 and 2012 in $p p$ collisions with a centre-of-mass energy of 7 and $8 \mathrm{TeV}$, respectively.

2. $B_{(s)}^{0} \rightarrow e^{+} \mu^{-}$

$B^{0}$ and $B_{s}^{0}$ meson decays to an electron and a muon provide the simplest experimental signature at $\mathrm{LHCb}$ for a process that violates lepton flavour conservation.

The events are triggered by muons having high transverse momentum or by electrons with large transverse energy, as measured by the muon detector and electromagnetic calorimeter, respectively. The electron and the muon tracks, identified via muon, calorimeter and Cherenkov detector informations combined, are required to form a good quality secondary vertex in order to reject the large amount of tracks promptly produced in the $p p$ collisions. The momentum of the electron, as measured by the tracking system, is corrected for bremsstrahlung losses by adding energy deposits in the electromagnetic calorimeter, if found to be consistent with photons emitted before the magnet. Since this energy is successfully recovered in only about $60 \%$ of the candidates, the remaining $40 \%$ are analysed in a separate category, as they exhibit a sizeably worse invariant mass resolution.

To reduce the combinatorial background, mostly originating from genuine electrons and muons from semileptonic decays of 2 distinct $B_{(s)}^{0}$ mesons, a Boosted Decision Tree (BDT) algorithm is trained on simulated signal events and, as a proxy for the background, on same-sign $e^{ \pm} \mu^{ \pm}$events.

\footnotetext{
${ }^{1}$ Charge conjugation is implied in this document unless otherwise stated.
} 
The events are categorised in bins of increasing sensitivity in the BDT response.

The number of signal events is converted into a branching fraction by using two normalisation channels: $B^{0} \rightarrow K^{+} \pi^{-}$and $B^{+} \rightarrow J / \psi\left(\mu^{+} \mu^{-}\right) K^{+}$, having signal-like reconstruction and trigger efficiencies, respectively. The signal branching fraction is thus computed as the efficiency corrected ratio of yields with respect to the normalisation channels, and by using their known branching fraction as an external input, according to:

$$
\mathcal{B}\left(B_{(s)}^{0} \rightarrow e^{+} \mu^{-}\right)=\frac{N_{B_{(s)}^{0} \rightarrow e^{+} \mu^{-}}}{N_{\text {norm }}} \times \frac{\varepsilon_{\text {norm }}}{\varepsilon_{\text {sig }}} \times \mathcal{B}_{\text {norm }} \times \frac{f_{d}}{f_{d(s)}} .
$$

The last factor in Eq. 2.1 accounts for the different hadronisation rates of $B_{s}^{0}$ and $B^{(0)+}$ mesons ( $f_{u}=f_{d}$ is assumed) by using the LHCb measurement $f_{s} / f_{d}=0.259 \pm 0.015$ [10].

After the full selection, two classes of background decays still pollute the signal: $B_{(s)}^{0} \rightarrow h^{+} h^{\prime-}$, when the two hadrons are misidentified as an electron and a muon, and semileptonic $b$-hadron decays. Both these contributions are evaluated from data-calibrated simulated events by normalising to the $B^{+} \rightarrow J / \psi\left(\mu^{+} \mu^{-}\right) K^{+}$yield. Since the $B_{(s)}^{0} \rightarrow h^{+} h^{--}$background directly peaks under the signal, special attention has been put in choosing a particle identification requirement that suppresses its level to a negligible amount, while retaining most of the signal. An independent and fully data-driven estimate also confirms that this background contribution is negligible. Among semileptonic decays, only $B^{0} \rightarrow \pi^{+} \mu^{-} \bar{v}_{\mu}$ and $\Lambda_{b}^{0} \rightarrow p \mu^{-} \bar{v}_{\mu}$ are found to survive the signal selection and are therefore included as separate components in the mass fit.

The number of signal candidates is extracted from an invariant mass fit performed simultaneously in the two bremsstrahlung categories and the BDT bins in which the events are split. Fig. 1 shows the results in the high BDT region, where the signal sensitivity is higher. Since no signal is ob-

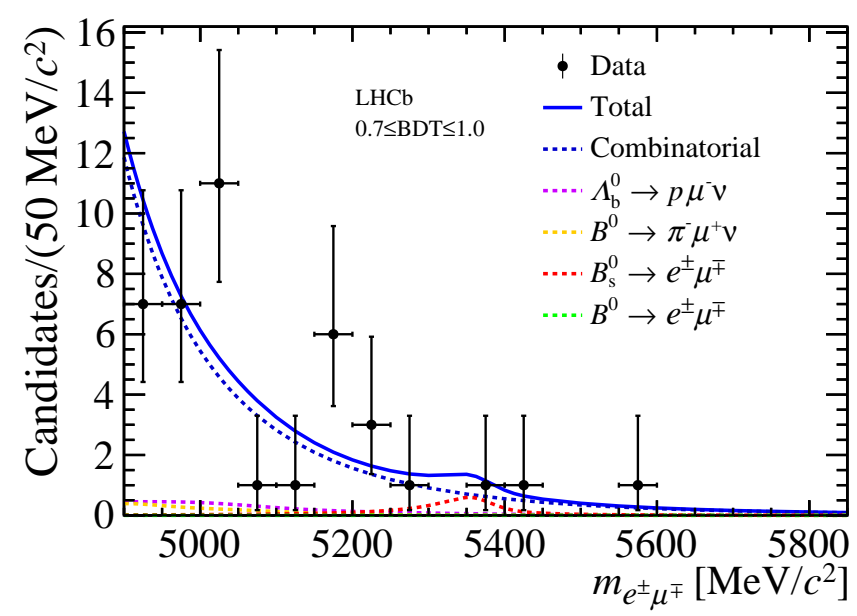

Figure 1: Invariant mass fit to the $B_{(s)}^{0} \rightarrow e^{+} \mu^{-}$candidates in the high sensitivity region.

served, upper limits on the branching fractions are computed according to the $\mathrm{CL}_{s}$ method [11], yielding [12]:

$$
\begin{aligned}
& \mathcal{B}\left(B_{s}^{0} \rightarrow e^{+} \mu^{-}\right)<6.3(5.4) \times 10^{-9}, \\
& \mathcal{B}\left(B^{0} \rightarrow e^{+} \mu^{-}\right)<1.3(1.0) \times 10^{-9}
\end{aligned}
$$


at $95 \%(90 \%) \mathrm{CL}$, under the assumption that the $B_{s}^{0}$ decay amplitude is entirely dominated by the heavy mass eigenstate. Since the sizeable width difference among the $B_{s}^{0}$ mass eigenstates implies different detector acceptances, the limit has also been computed under the opposite assumption of a decay fully dominated by the light mass eigenstate: $\mathcal{B}\left(B_{s} \rightarrow e^{+} \mu^{-}\right)<7.6(6.0) \times 10^{-9}$ at $95 \%$ (90\%) CL.

These results improve by a factor 2 to 3 the previous limits from $\mathrm{LHCb}$ [13].

3. $B_{(s)}^{0} \rightarrow \tau^{+} \mu^{-}$

Two other LFV channels accessible at LHCb are $B^{0} \rightarrow \tau^{+} \mu^{-}$and $B_{s}^{0} \rightarrow \tau^{+} \mu^{-}$, where the $\tau$ leptons are reconstructed in $\tau^{-} \rightarrow \pi^{-} \pi^{+} \pi^{-} \nu_{\tau}$ decays, allowing for a precise vertex reconstruction. Signal candidates are triggered by muons with high transverse momentum, and at least two reconstructed tracks are required to form a well detached vertex. Since the presence of a neutrino in the final state prevents the computation of the $B_{(s)}^{0}$ invariant mass, kinematic constraints are applied to the decay chain to reconstruct the mass analytically. This reconstructed mass allows to remove a large portion of the background without affecting the signal, as determined on simulated signal events and same-sign $\tau^{ \pm} \mu^{ \pm}$data.

A BDT algorithm is trained on simulated signal events against same-sign data, by exploiting track isolation variables, to reduce the combinatorial background to negligible amounts. Partially reconstructed events like $B_{(s)}^{0} \rightarrow D_{(s)}^{-}\left(\mu^{-} \bar{v}_{\mu}\right) \pi^{+} \pi^{-} \pi^{+}$have signal-like topology, but a requirement on the reconstructed $\tau$ decay time removes this component since, conversely to the signal, the three charged pions originate directly from the $B_{(s)}^{0}$ vertex. On the other hand, partially reconstructed semileptonic $B$ decays in which the $D_{(s)}$ meson decays hadronically, represent an irreducible background component that is therefore included in the mass fit. The normalisation channel
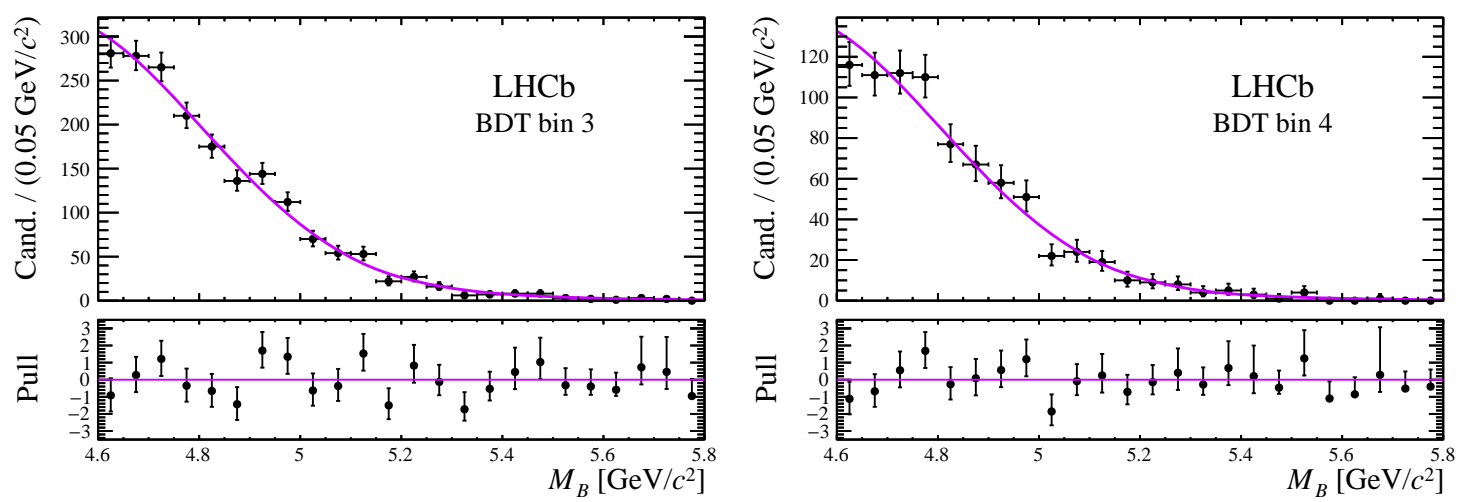

Figure 2: Invariant mass spectra of $B_{(s)}^{0} \rightarrow \tau^{+} \mu^{-}$candidates in the two BDT bins with the highest sensitivity. The fit projections under the hypothesis of $B_{s}^{0}$ signal only are overlaid.

$B^{0} \rightarrow D^{-}\left(K^{+} \pi^{-} \pi^{-}\right) \pi^{+}$is exploited to convert the observed signal yield into a branching fraction, equivalently to Eq. 2.1. This channel has an abundant yield and a similar topology to the signal, but the events are triggered by the hadrons: both the signal and normalisation trigger efficiencies are therefore evaluated on high-purity data control samples.

A second BDT algorithm is employed to categorise the signal candidates in bins of increasing 
sensitivity by exploiting event kinematics and in particular the masses of the three-pion system and of the two combinations of oppositely charged pions. These two variables provide good separation power against background as $\tau$ decays mainly proceed through $a_{1}(1260) \rightarrow \pi^{+} \pi^{-} \pi^{-}$and $\rho(770)^{0} \rightarrow \pi^{+} \pi^{-}$resonances [14].

The reconstructed mass of the selected candidates (Fig. 2) shows no evidence of signal excess: an upper limit on the branching fractions is computed according to the $\mathrm{CL}_{s}$ method at $95 \%$ (90\%) CL [15]:

$$
\begin{aligned}
& \mathcal{B}\left(B_{s}^{0} \rightarrow \tau^{+} \mu^{-}\right)<4.2(3.4) \times 10^{-5}, \\
& \mathcal{B}\left(B^{0} \rightarrow \tau^{+} \mu^{-}\right)<1.4(1.2) \times 10^{-5},
\end{aligned}
$$

where the two limits are separately derived from mass fits under the hypotheses of $B_{s}^{0}$-only and $B^{0}$-only signal, since their overlap is significant.

These limits represent the first measurement on the $B_{s}^{0}$ mode and an improvement by a factor of $\sim 2$ with respect to the limit set by BaBar on the $B^{0}$ mode [16].

4. $B^{+} \rightarrow K^{+} e^{ \pm} \mu^{\mp}$

$B^{+} \rightarrow K^{+} e^{ \pm} \mu^{\mp}$ decays require the reconstruction of an additional kaon track with respect to $B_{(s)}^{0} \rightarrow e^{+} \mu^{-}$, but are less affected by backgrounds and therefore represent a sensitive channel to search for LFV at LHCb.

Signal events are triggered by muons having high transverse momentum and by requiring at least two tracks having a significant displacement with respect to the interaction vertex. With the same criteria, $B^{+} \rightarrow J / \psi\left(\mu^{+} \mu^{-}\right) K^{+}$decays are selected for the normalisation, due to their large abundance and signal-like characteristics.

Double semileptonic decays like $B^{+} \rightarrow \bar{D}^{0}\left(K^{+} Y l^{-} \bar{v}_{l}\right) X l^{+} v_{l}$, where $X$ and $Y$ are hadrons, are rejected by imposing $m\left(K^{+} l^{-}\right)>1885 \mathrm{MeV} / c^{2}$, while decays involving charmonium resonances are removed via appropriate mass vetoes. Fully- and partially-reconstructed backgrounds, in which at least one particle in the final state is misidentified, are strongly suppressed by means of PID requirements as well as a dedicated BDT classifier, trained on simulated signal events versus lowmass data sidebands. A second BDT, based on $B$ kinematics and track isolation criteria, is trained on the high-mass data sidebands to reduce the combinatorial background.

The resulting mass spectra and fit functions are shown in Fig. 3, where the two charge combinations are analysed separately since NP might affect them in different ways. The selected candidates are consistent with the background-only hypothesis, therefore upper limits on the branching fractions are computed with the $\mathrm{CL}_{s}$ method at $95 \%$ (90\%) CL [17] ${ }^{2}$ :

$$
\begin{aligned}
& \mathcal{B}\left(B^{+} \rightarrow K^{+} \mu^{-} e^{+}\right)<9.5(7.0) \times 10^{-9}, \\
& \mathcal{B}\left(B^{+} \rightarrow K^{+} \mu^{+} e^{-}\right)<8.8(6.4) \times 10^{-9} .
\end{aligned}
$$

The results improve the limits previously set by the BaBar experiment [18] by an order of magnitude.

\footnotetext{
${ }^{2}$ The $B^{+} \rightarrow K^{+} e^{ \pm} \mu^{\mp}$ results reported in this Section represent an update of the preliminary results shown at the conference.
} 

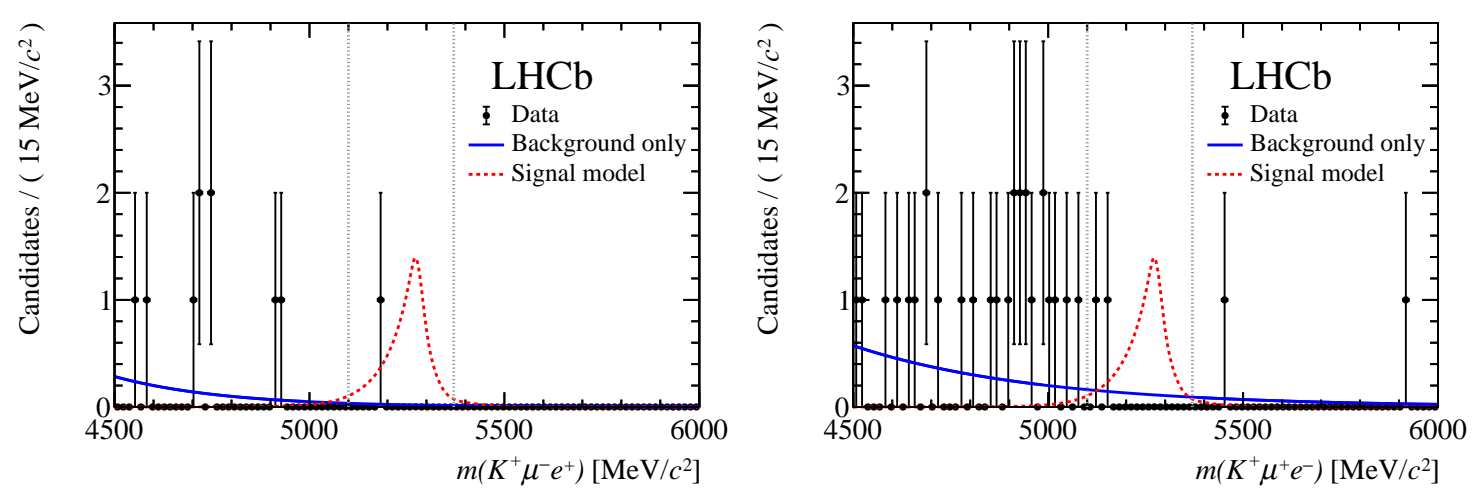

Figure 3: Invariant mass spectra of $B^{+} \rightarrow K^{+} e^{ \pm} \mu^{\mp}$ candidates with the background (blue) and signal (red) distributions superimposed. The signal distribution is arbitrarily normalised to 10 events.

\section{Conclusions}

Several deviations from the universality of the lepton couplings in $b$-hadron decays hint at possible LFV. Three recent searches performed at LHCb have been reported in this document, resulting in the most stringent limits to $B_{(s)}^{0} \rightarrow e^{+} \mu^{-}, B_{(s)}^{0} \rightarrow \tau^{+} \mu^{-}$and $B^{+} \rightarrow K^{+} e^{ \pm} \mu^{\mp}$ decays to date. New Physics model parameters are consequently narrowed down in the light of these results $[19,20]$ : as an example, Fig. 4 shows how the $B_{(s)}^{0} \rightarrow e^{+} \mu^{-}$limits are translated into lower bounds on the LQ masses in the Pati-Salam model [21], with values, above $100 \mathrm{TeV} / \mathrm{c}^{2}$, which are out of reach for direct searches at the LHC.
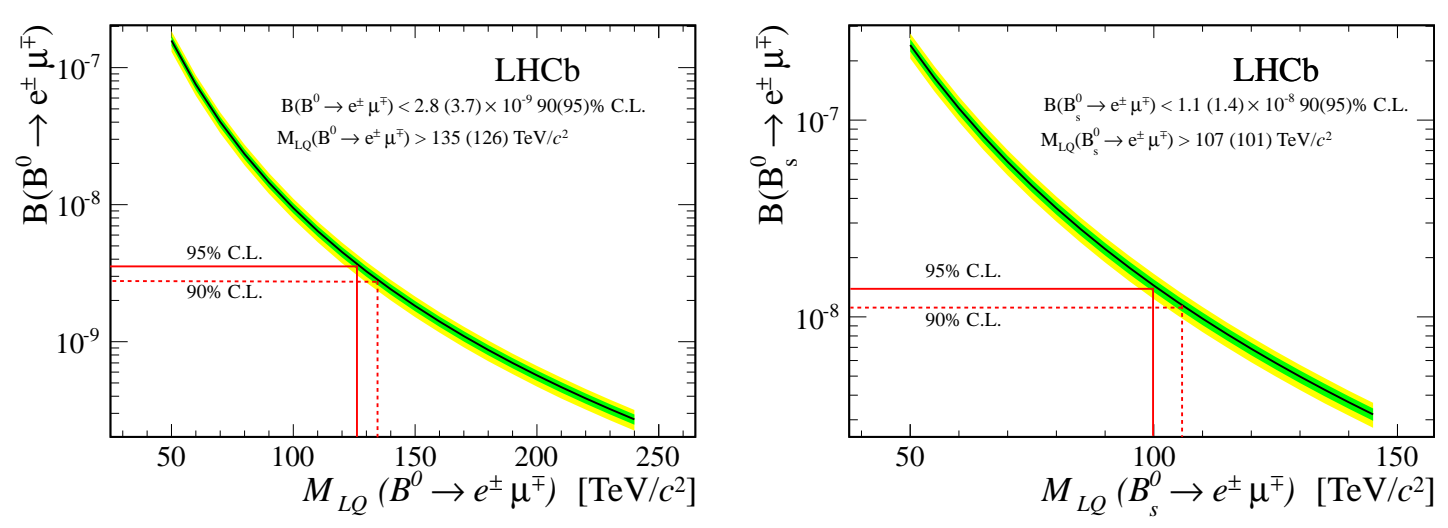

Figure 4: Branching fraction of $B^{0} \rightarrow e^{+} \mu^{-}$(left) and $B_{s}^{0} \rightarrow e^{+} \mu^{-}$(right) decays as a function of the leptoquark mass. The limit values (red lines) represent the LHCb result from [13].

\section{References}

[1] MEG collaboration, Search for the lepton flavour violating decay $\mu^{+} \rightarrow \mathrm{e}^{+} \gamma$ with the full dataset of the MEG experiment, Eur. Phys. J. C76 (2016) 434 [1605.05081]. 
[2] SINDRUM collaboration, Search for the Decay $\mu^{+} \rightarrow e^{+} e^{+} e^{-}$, Nucl. Phys. B299 (1988) 1.

[3] SINDRUM II collaboration, A Search for muon to electron conversion in muonic gold, Eur. Phys. J. C47 (2006) 337.

[4] L. Calibbi and G. Signorelli, Charged Lepton Flavour Violation: An Experimental and Theoretical Introduction, Riv. Nuovo Cim. 41 (2018) 71 [1709.00294].

[5] J. Aebischer, W. Altmannshofer, D. Guadagnoli, M. Reboud, P. Stangl and D. M. Straub, B-decay discrepancies after Moriond 2019, 1903.10434.

[6] A. Crivellin, L. Hofer, J. Matias, U. Nierste, S. Pokorski and J. Rosiek, Lepton-flavour violating B decays in generic Z' models, Phys. Rev. D92 (2015) 054013 [1504.07928].

[7] G. Hiller, D. Loose and K. Schönwald, Leptoquark Flavor Patterns \& B Decay Anomalies, JHEP 12 (2016) 027 [1609.08895].

[8] S. L. Glashow, D. Guadagnoli and K. Lane, Lepton Flavor Violation in B Decays?, Phys. Rev. Lett. 114 (2015) 091801 [1411.0565].

[9] LHCB collaboration, The LHCb Detector at the LHC, JINST 3 (2008) S08005.

[10] LHCB collaboration, Updated average $f_{s} / f_{d}$ b-hadron production fraction ratio for $7 \mathrm{TeV} p p$ collisions, .

[11] A. L. Read, Presentation of search results: The CL(s) technique, J. Phys. G28 (2002) 2693.

[12] LHCB collaboration, Search for the lepton-flavour violating decays $B_{(s)}^{0} \rightarrow e^{ \pm} \mu^{\mp}, J H E P 03$ (2018) 078 [1710.04111].

[13] LHCB collaboration, Search for the lepton-flavor violating decays $B_{s}^{0} \rightarrow e^{ \pm} \mu^{\mp}$ and $B^{0} \rightarrow e^{ \pm} \mu^{\mp}$, Phys. Rev. Lett. 111 (2013) 141801 [1307.4889].

[14] ALEPH collaboration, Branching ratios and spectral functions of tau decays: Final ALEPH measurements and physics implications, Phys. Rept. 421 (2005) 191 [hep-ex/0506072].

[15] LHCB collaboration, Search for the lepton-flavour-violating decays $B_{s}^{0} \rightarrow \tau^{ \pm} \mu^{\mp}$ and $B^{0} \rightarrow \tau^{ \pm} \mu^{\mp}$, 1905.06614.

[16] BABAR collaboration, Searches for the decays $B^{0} \rightarrow \ell^{ \pm} \tau^{\mp}$ and $B^{+} \rightarrow \ell^{+} v(l=e, \mu)$ using hadronic tag reconstruction, Phys. Rev. D77 (2008) 091104 [0801.0697].

[17] LHCB collaboration, Search for the lepton-flavour violating decays $B^{+} \rightarrow K^{+} \mu^{ \pm} e^{\mp}, 1909.01010$.

[18] BABAR collaboration, Measurements of branching fractions, rate asymmetries, and angular distributions in the rare decays $B \rightarrow K \ell^{+} \ell^{-}$and $B \rightarrow K^{*} \ell^{+} \ell^{-}$, Phys. Rev. D73 (2006) 092001 [hep-ex/0604007].

[19] I. de Medeiros Varzielas and G. Hiller, Clues for flavor from rare lepton and quark decays, JHEP 06 (2015) 072 [1503.01084].

[20] C. Cornella, J. Fuentes-Martin and G. Isidori, Revisiting the vector leptoquark explanation of the B-physics anomalies, JHEP 07 (2019) 168 [1903.11517].

[21] G. Valencia and S. Willenbrock, Quark - lepton unification and rare meson decays, Phys. Rev. D50 (1994) 6843 [hep-ph/9409201]. 